Published in final edited form as:

Nature. 2002 December 19; 420(6917): 860-867. doi:10.1038/nature01322.

\title{
Inflammation and cancer
}

\author{
Lisa M. Coussens ${ }^{\star}, \dagger, \S$ and Zena Werb $\ddagger, \S$ \\ Lisa M. Coussens: coussens@cc.ucsf.edu; Zena Werb: zena@itsa.ucsf.edu \\ * Cancer Research Institute, University of California, San Francisco, California 94143 USA \\ † Department of Pathology, University of California, San Francisco, California 94143 USA \\ ‡ Department of Anatomy, University of California, San Francisco, California 94143 USA \\ $\S$ UCSF Comprehensive Cancer Center, University of California, San Francisco, California 94143 \\ USA
}

\section{Abstract}

Recent data have expanded the concept that inflammation is a critical component of tumour progression. Many cancers arise from sites of infection, chronic irritation and inflammation. It is now becoming clear that the tumour microenvironment, which is largely orchestrated by inflammatory cells, is an indispensable participant in the neoplastic process, fostering proliferation, survival and migration. In addition, tumour cells have co-opted some of the signalling molecules of the innate immune system, such as selectins, chemokines and their receptors for invasion, migration and metastasis. These insights are fostering new anti-inflammatory therapeutic approaches to cancer development.

The functional relationship between inflammation and cancer is not new. In 1863, Virchow hypothesized that the origin of cancer was at sites of chronic inflammation, in part based on his hypothesis that some classes of irritants, together with the tissue injury and ensuing inflammation they cause, enhance cell proliferation ${ }^{1}$. Although it is now clear that proliferation of cells alone does not cause cancer, sustained cell proliferation in an environment rich in inflammatory cells, growth factors, activated stroma, and DNA-damage-promoting agents, certainly potentiates and/or promotes neoplastic risk. During tissue injury associated with wounding, cell proliferation is enhanced while the tissue regenerates; proliferation and inflammation subside after the assaulting agent is removed or the repair completed. In contrast, proliferating cells that sustain DNA damage and/or mutagenic assault (for example, initiated cells) continue to proliferate in microenvironments rich in inflammatory cells and growth/ survival factors that support their growth. In a sense, tumours act as wounds that fail to heal ${ }^{2}$.

Today, the causal relationship between inflammation, innate immunity and cancer is more widely accepted; however, many of the molecular and cellular mechanisms mediating this relationship remain unresolved - these are the focus of this review. Furthermore, tumour cells may usurp key mechanisms by which inflammation interfaces with cancers, to further their colonization of the host. Although the acquired immune response to cancer is intimately related to the inflammatory response, this topic is beyond the scope of this article, but readers are referred to several excellent reviews ${ }^{3,4}$.

\section{An overview of inflammation}

To understand the role of inflammation in the evolution of cancer, it is important to understand what inflammation is and how it contributes to physiological and pathological processes such as wound healing and infection (Fig. 1). In response to tissue injury, a multifactorial network 
of chemical signals initiate and maintain a host response designed to 'heal' the afflicted tissue. This involves activation and directed migration of leukocytes (neutrophils, monocytes and eosinophils) from the venous system to sites of damage (Box 1), and tissue mast cells also have a significant role. For neutrophils, a four-step mechanism is believed to coordinate recruitment of these inflammatory cells to sites of tissue injury and to the provisional extracellular matrix (ECM) that forms a scaffolding upon which fibroblast and endothelial cells proliferate and migrate, thus providing a nidus for reconstitution of the normal microenvironment ${ }^{5}$. These steps involve: activation of members of the selectin family of adhesion molecules (L- P-, and E-selectin) that facilitate rolling along the vascular endothelium; triggering of signals that activate and upregulate leukocyte integrins mediated by cytokines and leukocyte-activating molecules; immobilization of neutrophils on the surface of the vascular endothelium by means of tight adhesion through $\alpha_{4} \beta_{1}$ and $\alpha_{4} \beta_{7}$ integrins binding to endothelial vascular cell-adhesion molecule-1 (VCAM-1) and MadCAM-1, respectively; and transmigration through the endothelium to sites of injury, presumably facilitated by extracellular proteases, such as matrix metalloproteinases (MMPs).

\section{Box 1}

\section{Wound healing as an example of physiological inflammation}

\section{Cellular components}

Platelet activation and aggregation, in addition to accelerating coagulation, provide a bolus of secreted proteins and $\alpha$-granule contents to the immediate area, all of which help initiate and accelerate the inflammatory response by the host. Examples of such secreted proteins include arachodonic acid metabolites, heparin, serotonin, thrombin, coagulation factors (factor V), adhesive proteins (fibrinogen and von Willebrand factor), plasma proteins (immunoglobulin- $\gamma$ and albumin), cell growth factors (platelet-derived growth factor (PDGF), platelet-derived angiogenesis factor, transforming growth factor- $\alpha$ (TGF- $\alpha$ ), TGF$\beta$ and basic fibroblast growth factor (bFGF)), enzymes (heparanase and factor XIII) and protease inhibitors (plasminogen activator inhibitor-1, $\alpha 2$-macroglobulin and $\alpha 2$ antiplasmin). Following platelet-induced haemostasis and release of TGF- $\beta 1$ and PDGF, formation of granulation tissue is facilitated by chemotaxis of neutrophils, monocytes, fibroblasts and myofibroblasts, as well as by synthesis of new extracellular matrix (ECM) and neoangiogenesis.

Neutrophil chemotaxis is stimulated by factors such as circulating complement factor 5 (C5a), leukotriene B4, kallikrein, bacterial products (if present) and numerous factors released from platelets at the site (for example, PDGF, TGF- $\beta$, platelet-activating factor and platelet factor-4 (PF-4)). Although terminally differentiated with little biosynthetic machinery, neutrophils are capable of considerable production of cytokines/chemokines necessary for effector cell recruitment, activation and response15. These phagocytic cells initiate wound healing by serving as a source of early-response pro-inflammatory cytokines such as tumour necrosis factor- $\alpha$ (TNF- $\alpha) 68$, and interleukin (IL)- $1 \alpha$ and IL-1 $1 \beta 69$. These cytokines mediate leukocyte adherence to the vascular endothelium, thus targeting and restricting leukocytes to areas of repair, and initiate repair by inducing expression of matrix metalloproteinases (MMPs) and keratinocyte growth factor (KGF/FGF-7) by fibroblasts70.

In response to tissue injury, mononuclear phagocytes (that is, macrophage progenitors) migrate from the venous system to the site of tissue injury. They are guided to the site by chemotactic factors, including PF-4, TGF- $\beta$, PDGF, chemokines (monocyte chemoattractant protein-1, -2 and -3 (MCP-1/CCL2, MCP-2/CCL8 and MCP-3/CCL7), macrophage inflammatory protein- $1 \alpha$ and $-1 \beta$ (MIP- $1 \alpha /$ CCL 3 and MIP-1 $\beta / C C L 4)$, and the cytokines IL-1 $\beta$ and TNF- $\alpha$. Deployment of monocytes/macrophages to the site of injury 
peaks as the number of neutrophils decline. Once present, however, they differentiate into mature macrophages or immature dendritic cells71. After activation, macrophages are the main source of growth factors and cytokines (TGF- $\beta 1$, PDGF, bFGF, TGF- $\alpha$, insulin-like growth factor (IGF)-I and -II, TNF- $\alpha$ and IL-1) that modulate tissue repair. Cells in their local microenvironment (for example, endothelial, epithelial, mesenchymal or neuroendocrine cells) are profoundly affected by macrophage products. Macrophages also regulate local tissue remodelling by inducing ECM components, stimulating production of proteolytic enzymes (for example, MMPs and urokinase-type plasminogen activator (uPA)), clearing apoptotic and necrotic cells, and modulating angiogenesis through local production of thrombospondin-1 (refs 72, 73).

Following their activation, mast cells are full of stored and newly synthesized inflammatory mediators. This cell type synthesizes and stores histamine, cytokines and proteases complexed to highly sulphated proteoglycans within granules, and produces lipid mediators and cytokines upon stimulation. Once activated by complement or by binding of antigens to immunoglobulin $\mathrm{E}$ ( $\mathrm{IgE}$ ) bound to high-affinity $\mathrm{IgE}$ receptors (FceRI), they degranulate, releasing mediators including heparin, heparanase, histamine, MMPs and serine proteases, and various polypeptide growth factors, including bFGF and vascular endothelial growth factor $^{74}$. These function both in the early initiation phase of inflammation (for example, vascular reaction and exudation), and in the late phase where leukocyte accumulation and wound healing takes place.

\section{Chemotactic cytokines}

Chemokines are classified into polypeptide groups identified by the location of cysteine residues near their amino termini (for example, $\mathrm{C}-\mathrm{C}, \mathrm{C}-\mathrm{X}-\mathrm{C}, \mathrm{C}$ and $\mathrm{CX}_{3} \mathrm{C}$ ). Chemokines represent the largest family of cytokines ( 41 human members), forming a complex network for the chemotactic activation of all leukocytes. Chemokine receptors, members of the seven-transmembrane-spanning G-protein-coupled receptors, vary by cell type and degree of cell activation ${ }^{6}$. There is considerable redundancy in chemokine-receptor interaction, as many ligands bind different receptors, or vice versa.

The composition of chemokines produced at sites of tissue wounding not only recruits downstream effector cells (as discussed above), but also dictates the natural evolution of immune reactivity. For example, MCP-1/CCL2, a potent chemotactic protein for monocytes and lymphocytes, simultaneously induces expression of lymphocyte-derived IL-4 in response to antigen challenge while decreasing expression of IL-12 (ref. 75). The net effect of this alteration facilitates a switch from a $\mathrm{T}_{\mathrm{H}}$ 1-type to a $\mathrm{T}_{\mathrm{H}^{2}}$-type inflammatory response.

\section{Tissue repair}

In response to wounding, fibroblasts migrate into the wound bed and initially secrete collagen type III, which is later replaced by collagen type I. Synthesis and deposition of these collagens by fibroblasts is stimulated by factors including TGF- $\beta 1,-\beta 2$ and $-\beta 3$, PDGF, IL- $1 \alpha,-1 \beta$ and -4 , and mast cell tryptase. Once sufficient collagen has been generated, its synthesis is stopped; thus, during wound repair, production as well as the degradation of collagens is under precise spatial and temporal control.

The final phase of the healing process is re-epithelialization and migration of epithelial cells across this amalgam, in a process that requires both dissolution of the fibrin clot and degradation of the underlying dermal collagen. Epithelial cells at the leading edge of the wound express the UPA receptor, which is important for focal activation of UPA, and collagenolytic enzymes of the MMP family. In the absence of the fibrinolytic enzyme plasmin, derived from plasminogen after activation by uPA and tissue-PA, reepithelialization is dramatically delayed ${ }^{76}$. 
The pro-inflammatory properties of TGF- $\beta$, such as leukocyte recruitment, adhesion and regulation of MMP secretion and activation, are balanced by its ability to also reverse its role, and suppress these events and foster ECM synthesis to mediate tissue repair ${ }^{8}$. As inflammatory cells are activated, their complement of TGF- $\beta$ receptors change, resulting in differential susceptibility to TGF- $\beta$ and enhanced sensitivity to suppression by TGF- $\beta^{8}$, a critical event to resolving inflammation.

A family of chemotactic cytokines, named chemokines, which possess a relatively high degree of specificity for chemoattraction of specific leukocyte populations $1,6,7$, recruits downstream effector cells and dictates the natural evolution of the inflammatory response. The profile of cytokine/chemokines persisting at an inflammatory site is important in the development of chronic disease. The pro-inflammatory cytokine TNF- $\alpha$ (tumour necrosis factor- $\alpha$ ) controls inflammatory cell populations as well as mediating many of the other aspects of the inflammatory process. TGF- $\beta 1$ is also important, both positively and negatively influencing the processes of inflammation and repair ${ }^{8}$. The key concept is that normal inflammation - for example, inflammation associated with wound healing - is usually self-limiting; however, dysregulation of any of the converging factors can lead to abnormalities and ultimately, pathogenesis - this seems to be the case during neoplastic progression.

Neutrophils (and sometimes eosinophils) are the first recruited effectors of the acute inflammatory response. Monocytes, which differentiate into macrophages in tissues, are next to migrate to the site of tissue injury, guided by chemotactic factors. Once activated, macrophages are the main source of growth factors and cytokines, which profoundly affect endothelial, epithelial and mesenchymal cells in the local microenvironment. Mast cells are also important in acute inflammation owing to their release of stored and newly synthesized inflammatory mediators, such as histamine, cytokines and proteases complexed to highly sulphated proteoglycans, as well as lipid mediators.

\section{Inflammation and neoplastic progression}

Peyton Rous was the first to recognize that cancers develop from "subthreshold neoplastic states" caused by viral or chemical carcinogens that induce somatic changes 9,10 . These states, now known as 'initiation', involve DNA alterations, are irreversible and can persist in otherwise normal tissue indefinitely until the occurrence of a second type of stimulation (now referred to as 'promotion'). Promotion can result from exposure of initiated cells to chemical irritants, such as phorbol esters, factors released at the site of wounding, partial organ resection, hormones or chronic irritation and inflammation (Fig. 1). Functionally, many promoters, whether directly or indirectly, induce cell proliferation, recruit inflammatory cells, increase production of reactive oxygen species leading to oxidative DNA damage, and reduce DNA repair. Subversion of cell death and/or repair programmes occurs in chronically inflamed tissues, thus resulting in DNA replication and proliferation of cells that have lost normal growth control. Normal inflammation is self-limiting, because the production of anti-inflammatory cytokines follows the pro-inflammatory cytokines closely (Fig. 2). However, chronic inflammation seems to be due to persistence of the initiating factors or a failure of mechanisms required for resolving the inflammatory response. Why does the inflammatory response to tumours persist?

\section{Inflammatory cell component of tumours}

Tumour cells produce various cytokines and chemokines that attract leukocytes. The inflammatory component of a developing neoplasm may include a diverse leukocyte population - for example, neutrophils, dendritic cells, macrophages, eosinophils and mast cells, as well as lymphocytes - all of which are capable of producing an assorted array of 
cytokines, cytotoxic mediators including reactive oxygen species, serine and cysteine proteases, MMPs and membrane-perforating agents, and soluble mediators of cell killing, such as TNF- $\alpha$, interleukins and interferons (IFNs) ${ }^{11}, 12$.

Monocytes, in the presence of granulocyte-macrophage colony-stimulating factor (GM-CSF) and interleukin (IL)-4, differentiate into immature dendritic cells ${ }^{13}$. Dendritic cells migrate into inflamed peripheral tissue where they capture antigens and, after maturation, migrate to lymph nodes to stimulate T-lymphocyte activation. Soluble factors such as IL-6 and CSF-1, derived from neoplastic cells, push myeloid precursors towards a macrophage-like phenotype 14. Interestingly, dendritic cells found in neoplastic infiltrates are frequently immature and defective in T-cell stimulatory capacity.

Tumour-associated macrophages (TAMs) are a significant component of inflammatory infiltrates in neoplastic tissues and are derived from monocytes that are recruited largely by monocyte chemotactic protein (MCP) chemokines. TAMs have a dual role in neoplasms although they may kill neoplastic cells following activation by IL-2, interferon and IL-12 (refs 15,16 ), TAMs produce a number of potent angiogenic and lymphangiogenic growth factors, cytokines and proteases, all of which are mediators that potentiate neoplastic progression 17. TAMs and tumour cells also produce IL-10, which effectively blunts the anti-tumour response by cytotoxic T cells. During development of melanoma, activated macrophages produce TGF$\beta, \mathrm{TNF}-\alpha, \mathrm{IL}-1 \alpha$, arachidonate metabolites and extracellular proteases 18 . In response, melanocytes express IL-8 and vascular endothelial growth factor (VEGF)-A, thereby inducing vascular angiogenesis under paracrine control ${ }^{18}$. Indeed, macrophage infiltration is closely associated with the depth of invasion of primary melanoma due, in part, to macrophageregulated tumour-associated angiogenesis 19.

In addition to altering the local balance of pro-angiogenic factors during melanoma development, during human cervical carcinogenesis, TAMs express VEGF-C and VEGF-D as well as the VEGF receptor-3 (VEGFR-3), all of which are implicated in formation of lymphatic vessels and lymphatic metastases ${ }^{17}$. By placing TAMs at the centre of the recruitment and response to angiogenic and lymphangiogenic stimuli, they may foster the spread of tumours. TAMs also induce VCAM-1 expression on mesothelial cells, a step also believed to be key for tumour cell dissemination into the peritoneum 20 .

The functional significance of macrophage recruitment to sites of neoplastic growth has been examined by crossing transgenic mice expressing Polyoma virus middle T (PyMT) driven by the mouse mammary tumour virus (MMTV) long terminal repeat, which are prone to development of mammary cancer, with mice containing a null mutation in the CSF-1 gene $\left(C s f 1^{o p}\right)^{21}$. Whereas the absence of CSF-1 during early neoplastic development is without apparent consequence, development of late-stage invasive carcinoma and pulmonary metastases are significantly attenuated. The key difference between PyMT mice and PyMT/ $C s f 1^{o p} / C s f 1^{o p}$ mice is not in the apparent proliferative capacity of neoplastic epithelial cells, but in the failure to recruit mature macrophages into neoplastic tissue in the absence of CSF-1. Targeting CSF-1 expression specifically to mammary epithelium in CSF-1-null/PyMT mice restores macrophage recruitment, primary tumour development and metastatic potential ${ }^{12}$. A similar study showed that subcutaneous growth of Lewis lung cancer cells is impaired in $C s f 1^{o p} / C s f 1^{o p}$ mice22. In this example, however, tumours displayed a decreased mitotic index and pronounced necrosis, apparently resulting from diminished angiogenesis and impaired tumour-stroma formation. These defects were corrected by treatment of tumour-bearing mice with recombinant CSF-1 (ref. 22). Together, these genetic experiments provide a causal link between CSF-1-dependent infiltrating macrophages and the malignant potential of epithelial cells. 
Macrophages are not unique among inflammatory cells in potentiation of neoplastic processes. Genetic and functional experiments indicate that neutrophils, mast cells, eosinophils and activated $\mathrm{T}$ lymphocytes also contribute to malignancies by releasing extracellular proteases, pro-angiogenic factors and chemokines ${ }^{11,23-26}$.

\section{Cancers associated with chronic inflammation}

How are inflammatory cells co-opted into the neoplastic process? A plausible hypothesis is that many malignancies arise from areas of infection and inflammation, simply as part of the normal host response. Indeed, there is a growing body of evidence that many malignancies are initiated by infections ${ }^{11,27-29}$ (Table 1) - upwards of $15 \%$ of malignancies worldwide can be attributed to infections, a global total of 1.2 million cases per year ${ }^{11}$. Persistent infections within the host induce chronic inflammation. Leukocytes and other phagocytic cells induce DNA damage in proliferating cells, through their generation of reactive oxygen and nitrogen species that are produced normally by these cells to fight infection ${ }^{30}$. These species react to form peroxynitrite, a mutagenic agent ${ }^{30}$. Hence, repeated tissue damage and regeneration of tissue, in the presence of highly reactive nitrogen and oxygen species released from inflammatory cells, interacts with DNA in proliferating epithelium resulting in permanent genomic alterations such as point mutations, deletions, or rearrangements. Indeed, p53 mutations are seen at frequencies similar to those in tumours in chronic inflammatory diseases such as rheumatoid arthritis and inflammatory bowel disease ${ }^{31}$.

The strongest association of chronic inflammation with malignant diseases is in colon carcinogenesis arising in individuals with inflammatory bowel diseases, for example, chronic ulcerative colitis and Crohn's disease. Hepatitis C infection in the liver predisposes to liver carcinoma, an increased risk of bladder and colon carcinoma is associated with schistosomiasis, whereas chronic Helicobacter pylori infection is the world's leading cause of stomach cancer $^{32}$. The Gram-negative bacterium H. pylori is established as a definite carcinogen for the development of gastric cancer - the second most common type of cancer globally11,29 - and DNA damage resulting from chronic inflammation is believed the mechanism ${ }^{32}$. Exacerbating DNA damage induced by inflammatory cells is expression of macrophage migration inhibitory factor (MIF) from macrophages and T lymphocytes. MIF is a potent cytokine that overcomes 53 function by suppressing its transcriptional activity ${ }^{33}$. Chronic bypass of $\mathrm{p} 53$ regulatory functions in infiltrated tissues can enhance proliferation and extend life span, while also creating an environment with a deficient response to DNA damage, amplifying accumulation of potential oncogenic mutations.

Infectious viral agents, for example, DNA tumour viruses, may also directly transform cells by inserting active oncogenes into the host genome, although other mechanisms also are responsible. While many types of infectious agents are present in animals, only a subset of individuals infected with human papilloma virus, hepatitis B virus (HBV) or Epstein-Barr virus develop virus-associated malignancies. This may reflect immune suppression, the necessity of cofactors necessary for promotion or the fact that a neoplasm can develop only if viral infection has targeted a pluripotent progenitor or stem cell. Such stem cells are typically low in abundance and located in regions of tissues protected from agents that would otherwise harm them $^{34}$. In Rous sarcoma virus infections, inflammation is essential for tumour development and this requirement is mediated by factors such as TGF- $\beta$ and other cytokines produced by the inflammatory cells35. Epstein-Barr virus also causes sustained proliferation of B lymphocytes, which, when coupled with a secondary mutation, can result in neoplastic progression and malignant conversion to give rise to Burkett's lymphoma.

The molecular mechanism behind the associated risk of hepatocellular carcinoma resulting from $\mathrm{HBV}$ and/or hepatitis $\mathrm{C}$ virus (HCV) infection is uncertain. Although there is evidence for clonal integration of viral DNA in tumours and surrounding parenchyma cells, there are no 
defined transforming sequences found within the viral genomes that can act as viral oncogenes. Moreover, there is no evidence to suggest that viral integration activates either a classical cellular oncogene or inactivates a cellular tumour suppressor gene. HCV core protein interacts with the signal transducer and activator of transcription 3 (STAT3) protein ${ }^{36}$, a transcription factor involved in mediating cytokine signalling37. This interaction induces sustained phosphorylation of a critical tyrosine residue, resulting in enhanced proliferation and upregulation of $\mathrm{Bcl}-\mathrm{x}_{\mathrm{L}}$ and cyclin-D. Thus, chronic viral replication in hepatocytes may alter the local cytokine profile and the apoptotic or proliferative responses in infected cells, with an immune response to the viral proteins resulting in a state of chronic inflammation. Interestingly, a similar pathway involving inflammation, IL-6 and STAT3 is downstream of $H$. pylori in the generation of stomach cancer ${ }^{38}$.

\section{The chemokine connection}

Chemokines were initially defined functionally as soluble factors regulating directional migration of leukocytes during states of inflammation; however, chemokine biology extends to all cell types, including most human neoplastic cells ${ }^{6}$. Attention first focused on the role of chemokines during malignancy when it was reported that experimental animals without $\mathrm{T}$ or natural killer (NK) cell functions, when challenged with a tumour, showed a typical inflammatory infiltrate; this suggested that neoplastic cells either produce chemotactic factors or induce their expression in nearby 'host' cells ${ }^{39}$. It is now appreciated that the chemokinereceptor system can be altered dramatically in neoplastic tissue, particularly at the invasive edges. Moreover, chemokines induce direct effects on stromal and neoplastic cells in addition to their roles in regulating leukocyte recruitment (Fig. 2).

Regulation of tumour growth by chemokines-Some tumour cells not only regulate their chemokine expression to help recruit inflammatory cells, but also use these factors to further the tumour growth and progression. Melanoma is perhaps the best exemplar in which chemokines (for example, GRO $\alpha /$ CXCL1, GRO $\beta / C X C L 2$, GRO $\gamma / C X C L 3$ and IL-8/CXCL8) have been shown to exert autocrine control over neoplastic cell proliferation ${ }^{40}$. Blocking GRO $\alpha$ or the CXCR2 receptor attenuates melanoma cell proliferation in vitro ${ }^{41}$, whereas overexpression of GRO $\alpha$, GRO $\beta$ or GRO $\gamma$ in a variety of tumour-derived cell lines enhances their colony-forming activity and tumorigenicity in nude mice ${ }^{42,43}$. Other CXCR2 ligands have been identified as having autocrine roles in the growth of pancreatic, head and neck, and non-small-cell lung carcinoma44,45, whereas in mouse models, ENA-78/CXCL5 variably affects tumour growth, vascularity and apoptosis ${ }^{46}$. Macrophage pro-inflammatory chemokine-3 $\alpha$ (MIP-3 $\alpha /$ CCL20), a CC chemokine, is overexpressed in pancreatic carcinoma cells and infiltrating macrophages adjacent to tumours; MIP-3 $\alpha /$ CCL20 stimulates growth of neoplastic cells while simultaneously enhancing migration of $\mathrm{TAMs}^{47}$.

Regulation of angiogenesis by chemokines-Activation of angiogenic programmes represents a shift in the balance between pro- and anti-angiogenic factors48. Although angiogenesis is strictly controlled, it is associated with chronic inflammatory diseases, such as psoriasis, rheumatoid arthritis and fibrosis, as well as with tumour growth and metastasis ${ }^{48}$. It is well established that CXC chemokines with the three amino acids (Glu-Leu-Arg/ELR) immediately amino-terminal to the $\mathrm{CXC}$ motif $\left(\mathrm{ELR}^{+}\right)$are pro-angiogenic and stimulate endothelial cell chemotaxis, whereas ELR ${ }^{-}$CXC chemokines (for example, PF-4/CXCL4, MIG/CXCL9 and IP-10/CXCL10) possess angiostatic activities 44,49 . ELR ${ }^{+}$CXC ligands bind to CXCR2 and to a lesser degree to CXCR1, whereas ELR ${ }^{-}$CXC ligands bind to CXCR3, CXCR4 and CXCR5 (ref. 6). Compared to VEGF-A, murine MCP-5/CCL12 exhibits only modest mitogenic properties towards endothelial cells; however, it is a potent chemoattractant. In contrast, stromal-cell-derived factor 1 (SDF-1/CXCL12) induces endothelial expression of VEGF-A; VEGF-A in turn upregulates CXCR4 on endothelial cells7. Although it is not always 
clear if the angiostatic and angiogenic effects of chemokines are direct or indirect, it is accepted that the balance between the two regulates neoplastic cell physiology.

Chemokines and metastasis-Malignant cells that possess metastatic capacity have properties endowing them with the ability to invade and survive in ectopic tissue, venous and/ or lymphatic environments, as well as ability to reside and proliferate at a distal site (Fig. 3). Much debate exists as to whether malignant cells metastasize to environments favouring their specific growth or whether different organs are endowed with the ability to arrest or attract specific types of malignant cells through chemotactic factors (the so-called homing theory) 48. Studies using a mouse model by Muller and colleagues suggest that the pattern of breast cancer metastases is in part governed by specific interactions between CXCR4 and its ligand SDF-1/CXCL12 (ref. 50). CXCL12 is a rather unique chemokine in that it is the product of resting cells in multiple organs6, and is particularly highly expressed in target organs for breast cancer metastasis50. CXCL12 triggers chemotaxis of malignant mammary carcinoma cells in vitro, and the chemotactic activity of extracts of organs targeted by breast cancer cells (bone marrow, liver, lung and lymph nodes) can be neutralized by anti-CXCR4 antibodies. The involvement of CXCR4 in metastasis is not limited to breast cancer, as CXCR4 is expressed in tumour cell lines (for example, prostate carcinomas, B-cell lymphomas, astrogliomas and chronic lymphocytic leukaemias) that also respond to CXCL12 (ref. 51). The broader implications of these observations are that chemokines may be involved in regulating the spectrum of metastases in diverse cancer types.

\section{Tumours commandeer leukocyte adhesion mechanisms}

Tumour cells not only take advantage of the trophic factors made by inflammatory cells, but may also use the same adhesion molecules, chemokines and receptors to aid in migration and homing during distant metastatic spread. Evidence suggests that mechanisms used for homing of leukocytes may be appropriated for the dissemination of tumours via the bloodstream and lymphatics. Selectins are adhesion receptors that normally recognize certain vascular mucintype glycoproteins bearing the carbohydrate structure sialyl-Lewis $\mathrm{X}$ and facilitate leukocyte rolling along the blood vessels. Metastatic progression of many epithelial carcinomas correlates with tumour production of mucins containing sialyl-Lewis X. Lung colonization by melanoma cells that express sialyl-Lewis X is significantly reduced in E/P-selectin-deficient mice ${ }^{52}$. Pselectin deficiency attenuates tumour growth and metastasis, and tumours are significantly smaller in mice treated with a receptor antagonist peptide.

These results indicate that receptors expressed in the vasculature are crucial in targeting sialylLewis X-dependent cancer cells ${ }^{53}$. P-selectin facilitates human carcinoma metastasis in immunodeficient mice by mediating early interactions of platelets with blood-borne tumour cells via their cell-surface mucins, a process that can be blocked by heparin ${ }^{54}$. L-selectin on neutrophils, monocytes and/or NK cells also may facilitate metastasis ${ }^{55}$. Metastasis could involve the formation of tumour-platelet-leukocyte emboli that interact with the vasculature of distant organs. In addition, expression of L-selectin on tumour cells can foster metastasis to lymph nodes 56 .

\section{Inflammation as an anti-cancer therapeutic opportunity}

Perhaps the best evidence for the significance of inflammation during neoplastic progression comes from study of cancer risk among long-term users of aspirin and nonsteroidal antiinflammatory drugs (NSAIDs). Much data indicates that use of these drugs reduces colon cancer risk by $40-50 \%$, and may be preventative for lung, oesophagus and stomach cancer57,58. The ability of NSAIDs to inhibit cyclo-oxygenases (COX-1 and -2) underlies their mechanism(s) of chemoprevention. COX-2 converts arachidonic acid to prostaglandins, which in turn induces inflammatory reactions in damaged tissues59. Aspirin is non-selective 
in its inhibition of platelet function by acetylating and irreversibly inactivating both COX-1 and COX-2. Inactivation prevents platelet synthesis of prostaglandins, endoperoxides and thromboxane A2.

Other NSAIDs, for example, flurbiprofen, may have strong anti-metastatic effects because of their inhibition of platelet aggregation ${ }^{60}$. But NSAIDs may act through mechanisms other than inhibition of COX enzyme activity alone, as some NSAIDs lacking COX-inhibitory function show efficacy in inhibiting colon carcinogenesis ${ }^{61}$. Other mechanisms have been proposed $^{15}$, including induction of apoptosis through release of cytochrome $\mathrm{C}$ from mitochondria and subsequent activation of caspase- 9 and -3 , and/or interference with cell-cycle progression, reduction of carcinogen activation and stimulation of immune surveillance.

The pro-inflammatory cytokine TNF- $\alpha$ is also a key downstream mediator in inflammation. Despite the name, TNF- $\alpha$ is important in early events in tumours, regulating a cascade of cytokines, chemokines, adhesions, MMPs and pro-angiogenic activities ${ }^{1,62}$. Thus, TNF- $\alpha$ may be one of the ways in which inflammation acts as a tumour promoter. Blocking antibodies that have significant therapeutic efficacy in other inflammatory diseases ${ }^{63}$ may have applications in therapy in cancer.

Tumours are also rich in mucins and other ligands that may include the sialyl-Lewis X epitope recognized by selectins. Because selectins may have a role in metastasi ${ }^{54,55}$, targeting the selectin interaction with heparin or antagonists of the receptor may decrease metastasis ${ }^{54}$.

MMPs are produced by inflammatory cells and by stromal cells responding to chemokines and cytokines produced by inflammatory cells in tumour microenvironments ${ }^{25}$. Like inflammatory cells, MMPs may both promote tumour progression and attenuate it. Indeed, MMPs may mediate many of the actions of inflammatory cells in neoplasms ${ }^{64}$. MMPs can recruit inflammatory cells by releasing chemoattractants and motogens; they also generate growthpromoting and cytostatic signals. MMPs activate angiogenesis, but also produce fragments of basement-membrane collagens and plasminogen that are angiogenesis inhibitors. They have both apoptotic and anti-apoptotic actions. Thus, the efficacy of MMP inhibitors may be mediated, at least in part, through anti-inflammatory actions ${ }^{64,65}$. Given their diverse actions, it is also not surprising that trials with MMP inhibitors have had mixed results, with efficacy reported mostly during early tumour progression ${ }^{66}$.

\section{Inflammatory cells and cancer: friend or foe?}

It is now evident that inflammatory cells have powerful effects on tumour development. Early in the neoplastic process, these cells are powerful tumour promoters, producing an attractive environment for tumour growth, facilitating genomic instability and promoting angiogenesis. The inflammatory cells, and the chemokines and cytokines that they produce, influence the whole tumour organ, regulating the growth, migration and differentiation of all cell types in the tumour microenvironment, including neoplastic cells, fibroblasts and endothelial cells. Later in the tumorigenic process, neoplastic cells also divert inflammatory mechanisms such as selectin-ligand interactions, MMP production and chemokine functions to favour neoplastic spread and metastasis. This may be part of an attempt by the tumour to subvert immune cell functions, so favouring tumour development. Yet, the recruitment of inflammatory cells may also be counterproductive for tumour development, and also may represent an attempt by the host to suppress tumour growth.

The pro-tumour actions of inflammatory cells include releasing growth and survival factors, promoting angiogenesis and lymphangiogenesis, stimulating DNA damage, remodelling the ECM to facilitate invasion, coating tumour cells to make available receptors for disseminating cells via lymphatics and capillaries, and evading host defence mechanisms. Although 
inflammatory responses should also be anti-tumour, cancer patients are often defective in their inflammatory responses. This may arise by two distinct tumour-mediated mechanisms: a failure to upregulate the anti-inflammatory cytokines, or subversion of the host response resulting from desensitization of receptors owing to high chemokine and cytokine concentrations that then blunt systemic responses. Can we apply these new insights for targeting metastases?

It is clear that anti-inflammatory therapy is efficacious towards early neoplastic progression and malignant conversion. In a fully developed malignancy, there are 'excess' inflammatory cells in the tumour microenvironment. Does the tumour need inflammation to help foster angiogenesis? We must think globally and act locally. One approach is to evaluate whether functional polymorphisms in genes that regulate inflammatory processes (for example, genes encoding MMPs, cytokines, chemokines or selectins) harbour altered risk for developing cancer or are indicators of prognosis. Yet for all the local inflammation in tumours, in many cases the overall innate immunity of the host is blunted. The challenge for the future is to normalize the inflammatory network to regain a normal host response overall: decreasing the high levels of tumour-promoting properties of the infiltrating cells, such as pro-inflammatory cytokines, while increasing their tumour-suppressing properties, such as anti-inflammatory cytokines. In this way, later in tumour progression, we can harness the activities that are antitumour while suppressing those that are pro-tumour.

\section{Acknowledgments}

Supported by grants from the National Institutes of Health, the American Cancer Society, the V Foundation for Cancer Research, the Edward Mallinckrodt Jr Foundation for Medical Research, and the American Association for Cancer Research.

\section{References}

1. Balkwill F, Mantovani A. Inflammation and cancer: back to Virchow? Lancet 2001;357:539-545. [PubMed: 11229684]

2. Dvorak HF. Tumors: wounds that do not heal. Similarities between tumor stroma generation and wound healing. N Engl J Med 1986;315:1650-1659. [PubMed: 3537791]

3. Dranoff G. Tumour immunology: immune recognition and tumor protection. Curr Opin Immunol 2002;14:161-164.

4. Pardoll DM. Spinning molecular immunology into successful immunotherapy. Nature Rev Immunol 2002;2:227-238. [PubMed: 12001994]

5. Chettibi, S.; Ferguson, MWJ. Inflammation: Basic Principles and Clinical Correlates. Gallin, JI.; Snyderman, R., editors. Lipincott, Williams and Wilkinson; Philadelphia: 1999. p. 865-881.

6. Rossi D, Zlotnik A. The biology of chemokines and their receptors. Annu Rev Immunol 2000;18:217242. [PubMed: 10837058]

7. Homey B, Muller A, Zlotnik A. Chemokines: agents for the immunotherapy of cancer? Nature Rev Immunol 2002;2:175-184. [PubMed: 11913068]

8. Moustakas A, Pardali K, Gaal A, Heldin CH. Mechanisms of TGF- $\beta$ signaling in regulation of cell growth and differentiation. Immunol Lett 2002;82:85-91. [PubMed: 12008039]

9. Rous P, Kidd J. Conditional neoplasms and subthreshold neoplastic states: a study of the tar tumors of rabbits. J Exp Med 1941;73:365-389. [PubMed: 19871084]

10. Mackenzie IC, Rous P. The experimental disclosure of latent neoplastic changes in tarred skin. J Exp Med 1941;73:391-415. [PubMed: 19871085]

11. Kuper H, Adami HO, Trichopoulos D. Infections as a major preventable cause of human cancer. J Intern Med 2000;248:171-183. [PubMed: 10971784]

12. Wahl LM, Kleinman HK. Tumor-associated macrophages as targets for cancer therapy. J Natl Cancer Inst 1998;90:1583-1584. [PubMed: 9811301] 
13. Talmor M, et al. Generation of large numbers of immature and mature dendritic cells from rat bone marrow cultures. Eur J Immunol 1998;28:811-817. [PubMed: 9541575]

14. Allavena $P$, et al. The chemokine receptor switch paradigm and dendritic cell migration: its significance in tumor tissues. Immunol Rev 2000;177:141-149. [PubMed: 11138772]

15. Brigati C, Noonan DM, Albini A, Benelli R. Tumors and inflammatory infiltrates: friends or foes? Clin Exp Metastasis 2002;19:247-258. [PubMed: 12067205]

16. Tsung K, Dolan JP, Tsung YL, Norton JA. Macrophages as effector cells in interleukin 12-induced T cell-dependent tumor rejection. Cancer Res 2002;62:5069-5075. [PubMed: 12208763]

17. Schoppmann S, et al. Tumor-associated macrophages express lymphatic endothelial growth factors and are related to peritumoral lymphangiogenesis. Am J Pathol 2002;161:947-956. [PubMed: 12213723]

18. Torisu $\mathrm{H}$, et al. Macrophage infiltration correlates with tumor stage and angiogenesis in human malignant melanoma: possible involvement of TNF $\alpha$ and IL-1 $\alpha$. Int J Cancer 2000;85:182-188. [PubMed: 10629075]

19. Ono M, Torisu H, Fukushi J, Nishie A, Kuwano M. Biological implications of macrophage infiltration in human tumor angiogenesis. Cancer Chemother Pharmacol 1999;43:S69-S71. [PubMed: 10357562]

20. Jonjic N, et al. Expression of adhesion molecules and chemotactic cytokines in cultured human mesothelial cells. J Exp Med 1992;176:1165-1174. [PubMed: 1383376]

21. Lin EY, Nguyen AV, Russell RG, Pollard JW. Colony-stimulating factor 1 promotes progression of mammary tumors to malignancy. J Exp Med 2001;193:727-740. [PubMed: 11257139]

22. Nowicki A, et al. Impaired tumor growth in colony-stimulating factor 1 (CSF-1)-deficient, macrophage-deficient op/op mouse: evidence for a role of CSF-1-dependent macrophages in formation of tumor stroma. Int J Cancer 1996;65:112-119. [PubMed: 8543387]

23. DiCarlo E, et al. The intriguing role of polymorphonuclear neutrophils in antitumor reactions. Blood 2001;97:339-345. [PubMed: 11154206]

24. Coussens LM, et al. Inflammatory mast cells up-regulate angiogenesis during squamous epithelial carcinogenesis. Genes Dev 1999;13:1382-1397. [PubMed: 10364156]

25. Coussens LM, Tinkle CL, Hanahan D, Werb Z. MMP-9 supplied by bone marrow-derived cells contributes to skin carcinogenesis. Cell 2000;103:481-490. [PubMed: 11081634]

26. Bergers G, et al. Matrix metalloproteinase-9 triggers the angiogenic switch during carcinogenesis. Nature Cell Biol 2000;2:737-744. [PubMed: 11025665]

27. Blaser MJ, Chyou PH, Nomura A. Age at establishment of Helicobacter pylori infection and gastric carcinoma, gastric ulcer, and duodenal ulcer risk. Cancer Res 1995;55:562-565. [PubMed: 7834625]

28. Scholl SM, et al. Anti-colony-stimulating factor-1 antibody staining in primary breast adenocarcinomas correlates with marked inflammatory cell infiltrates and prognosis. J Natl Cancer Inst 1994;86:120-126. [PubMed: 8271294]

29. Shacter E, Weitzman SA. Chronic inflammation and cancer. Oncology 2002;16:217-226. [PubMed: 11866137]

30. Maeda H, Akaike T. Nitric oxide and oxygen radicals in infection, inflammation, and cancer. Biochemistry 1998;63:854-865. [PubMed: 9721338]

31. Yamanishi Y, et al. Regional analysis of p53 mutations in rheumatoid arthritis synovium. Proc Natl Acad Sci USA 2002;99:10025-10030. [PubMed: 12119414]

32. Ernst PB, Gold BD. The disease spectrum of Helicobacter pylori: the immunopathogenesis of gastroduodenal ulcer and gastric cancer. Annu Rev Microbiol 2000;54:615-640. [PubMed: 11018139]

33. Hudson JD, et al. A proinflammatory cytokine inhibits p53 tumor suppressor activity. J Exp Med 1999;190:1375-1382. [PubMed: 10562313]

34. Jensen UB, Lowell S, Watt FM. The spatial relationship between stem cells and their progeny in the basal layer of human epidermis: a new view based on whole-mount labeling and lineage analysis. Development 1999;126:2409-2418. [PubMed: 10226000] 
35. Martins-Green M, Boudreau N, Bissell MJ. Inflammation is responsible for the development of wound-induced tumors in chickens infected with Rous sarcoma virus. Cancer Res 1994;54:43344341. [PubMed: 7519120]

36. Yoshida T, et al. Activation of STAT3 by the hepatitis C virus core protein leads to cellular transformation. J Exp Med 2002;196:641-653. [PubMed: 12208879]

37. Bromberg J, Darnell JE. The role of STATs in transcriptional control and their impact on cellular function. Oncogene 2000;19:2468-2473. [PubMed: 10851045]

38. Tebbutt NC, et al. Reciprocal regulation of gastrointestinal homeostasis by SHP2 and STAT-mediated trefoil gene activation in gp130 mutant mice. Nature Med 2002;8:1089-1097. [PubMed: 12219085]

39. Mantovani A, Muzio M, Garlanda C, Sozzani S, Allavena P. Macrophage control of inflammation: negative pathways of regulation of inflammatory cytokines. Novartis Found Symp 2001;234:120 131. [PubMed: 11199092]

40. Richmond A, Thomas H. Purification of melanoma growth stimulatory activity. J Cell Physiol 1986;129:375-384. [PubMed: 3465735]

41. Norgauer J, Metzner B, Schraufstatter I. Expression and growth-promoting function of the IL-8 receptor $\beta$ in human melanoma cells. J Immunol 1996;156:1132-1137. [PubMed: 8557989]

42. Balentien E, Mufson BE, Shattuck RL, Derynck R, Richmond A. Effects of MGSA/GRO alpha on melanocyte transformation. Oncogene 1991;6:1115-1124. [PubMed: 1861861]

43. Owen JD, et al. Enhanced tumor-forming capacity for immortalized melanocytes expressing melanoma growth stimulatory activity/growth-regulated cytokine beta and gamma proteins. Int J Cancer 1997;73:94-103. [PubMed: 9334815]

44. Vicari AP, Caux C. Chemokines in cancer. Cytokine Growth Factor Rev 2002;13:143-154. [PubMed: 11900990]

45. Farrow B, Evers BM. Inflammation and the development of pancreatic cancer. Surg Oncol 2002;10:153-169. [PubMed: 12020670]

46. Arenberg DA, et al. Epithelial-neutrophil activating peptide (ENA-78) is an important angiogenic factor in non-small cell lung cancer. J Clin Invest 1998;102:465-472. [PubMed: 9691082]

47. Kleeff J, et al. Detection and localization of Mip-3 $\alpha /$ LARC/Exodus, a macrophage proinflammatory chemokine, and its CCR6 receptor in human pancreatic cancer. Int J Cancer 1999;81:650-657. [PubMed: 10225458]

48. Hanahan D, Weinberg RA. The hallmarks of cancer. Cell 2000;100:57-70. [PubMed: 10647931]

49. Strieter RM, et al. The functional role of the ELR motif in CXC chemokine-mediated angiogenesis. J Biol Chem 1995;270:27348-27357. [PubMed: 7592998]

50. Muller A, et al. Involvement of chemokine receptors in breast cancer metastasis. Nature 2001;410:5056. [PubMed: 11242036]

51. Moore MA. The role of chemoattraction in cancer metastases. BioEssays 2001;23:674-676. [PubMed: 11494314]

52. Kim YJ, Borsig L, Varki NM, Varki A. P-selectin deficiency attenuates tumor growth and metastasis. Proc Natl Acad Sci USA 1998;95:9325-9330. [PubMed: 9689079]

53. Zhang J, et al. Sialyl Lewis X-dependent lung colonization of B16 melanoma cells through a selectinlike endothelial receptor distinct from E- or P-selectin. Cancer Res 2002;62:4194-4198. [PubMed: 12154017]

54. Borsig L, et al. Heparin and cancer revisited: mechanistic connections involving platelets, P-selectin, carcinoma mucins, and tumor metastasis. Proc Natl Acad Sci USA 2001;98:3352-3357. [PubMed: 11248082]

55. Borsig L, Wong R, Hynes RO, Varki NM, Varki A. Synergistic effects of L- and P-selectin in facilitating tumor metastasis can involve non-mucin ligands and implicate leukocytes as enhancers of metastasis. Proc Natl Acad Sci USA 2002;99:2193-2198. [PubMed: 11854515]

56. Qian F, Hanahan D, Weissman IL. L-selectin can facilitate metastasis to lymph nodes in a transgenic mouse model of carcinogenesis. Proc Natl Acad Sci USA 2001;98:3976-3981. [PubMed: 11274419]

57. Baron JA, Sandler RS. Nonsteroidal anti-inflammatory drugs and cancer prevention. Annu Rev Med 2000;51:511-523. [PubMed: 10774479] 
58. Garcia-Rodriguez LA, Huerta-Alvarez C. Reduced risk of colorectal cancer among long-term users of aspirin and nonaspirin nonsteroidal antiinflammatory drugs. Epidemiology 2001;12:88-93. [PubMed: 11138826]

59. Williams CS, Mann M, DuBois RN. The role of cyclooxygenases in inflammation, cancer, and development. Oncogene 1999;18:7908-7916. [PubMed: 10630643]

60. Mamytbekova A, Rezabek K, Kacerovska H, Grimova J, Svobodova J. Antimetastatic effect of flurbiprofen and other platelet aggregation inhibitors. Neoplasma 1986;33:417-421. [PubMed: 3762804]

61. Elder DJ, Halton DE, Hague A, Paraskeva C. Induction of apoptotic cell death in human colorectal carcinoma cell lines by a cyclooxygenase-2 (COX-2)-selective nonsteroidal anti-inflammatory drug: independence from COX-2 protein expression. Clin Cancer Res 1997;3:1679-1683. [PubMed: 9815550]

62. Balkwill F. Tumor necrosis factor or tumor promoting factor? Cytokine Growth Factor Rev 2002;13:135-141. [PubMed: 11900989]

63. Shanahan JC, St Clair EW. Tumor necrosis factor-alpha blockade: a novel therapy for rheumatic disease. Clin Immunol 2002;103:231-242. [PubMed: 12173297]

64. Egeblad M, Werb Z. New functions for the matrix metalloproteinases in cancer progression. Nature Rev Cancer 2002;2:161-174. [PubMed: 11990853]

65. Overall CM, Lopez-Otin C. Strategies for MMP inhibition in cancer: innovations for the post-trial era. Nature Rev Cancer 2002;2:657-672. [PubMed: 12209155]

66. Coussens LM, Fingleton B, Matrisian LM. Matrix metalloproteinase inhibitors and cancer: trials and tribulations. Science 2002;295:2387-2392. [PubMed: 11923519]

67. Dalgleish AG, O'Byrne KJ. Chronic immune activation and inflammation in the pathogenesis of AIDS and cancer. Adv Cancer Res 2002;84:231-276. [PubMed: 11883529]

68. Feiken E, Romer J, Eriksen J, Lund LR. Neutrophils express tumor necrosis factor-alpha during mouse skin wound healing. J Invest Dermatol 1995;105:120-123. [PubMed: 7615965]

69. Hubner G, et al. Differential regulation of pro-inflammatory cytokines during wound healing in normal and glucocorticoid-treated mice. Cytokine 1996;8:548-556. [PubMed: 8891436]

70. Chedid M, Rubin JS, Csaky KG, Aaronson SA. Regulation of keratinocyte growth factor gene expression by interleukin 1. J Biol Chem 1994;269:10753-10757. [PubMed: 7511604]

71. Osusky R, Malik P, Ryan SJ. Retinal pigment epithelium cells promote the maturation of monocytes to macrophages in vitro. Ophthalmic Res 1997;29:31-36. [PubMed: 9112264]

72. DiPietro L. Wound healing: the role of the macrophage and other immune cells. Shock 1995;4:233240. [PubMed: 8564549]

73. Fritsch C, Simon-Assmann P, Kedinger M, Evans GS. Cytokines modulate fibroblast phenotype and epithelial-stroma interactions in rat intestine. Gastroenterology 1997;112:826-838. [PubMed: 9041244]

74. Grutzkau A, et al. Synthesis, storage, and release of vascular endothelial growth factor/vascular permeability factor (VEGF/VPF) by human mast cells: implications for the biological significance of VEGF206. Mol Biol Cell 1998;9:875-884. [PubMed: 9529385]

75. Chensue SW, Ruth JH, Warmington K, Lincoln P, Kunkel SL. In vivo regulation of macrophage IL-12 production during type 1 and type 2 cytokine-mediated granuloma formation. J Immunol 1995;155:3546-3551. [PubMed: 7561051]

76. Romer J, et al. Impaired wound healing in mice with a disrupted plasminogen gene. Nature Med 1996;2:287-292. [PubMed: 8612226] 
a

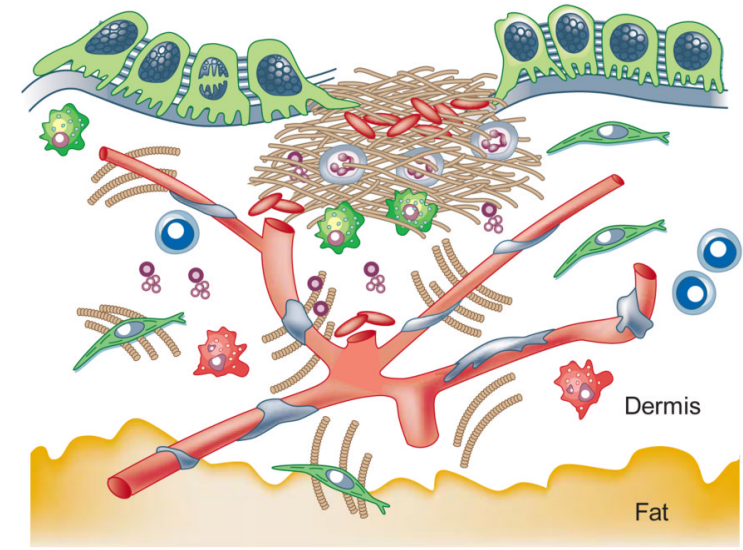

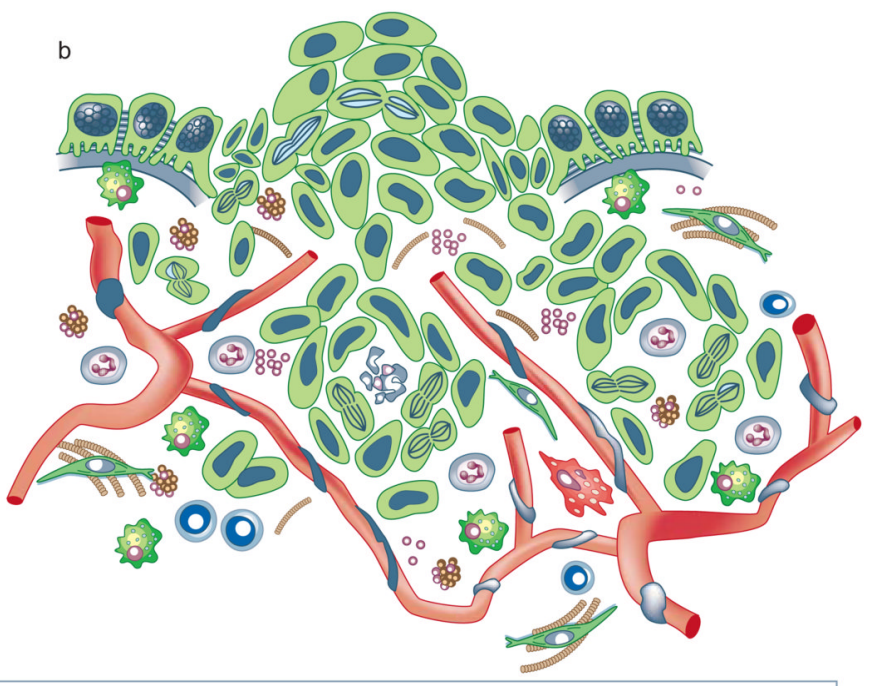

Endothelial cells and capillary support cells (pericytes, smooth muscle cells)

Mast cells/eosinophils/basophils Fibroblasts and fibrillar collagens

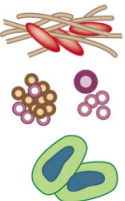

Platelets and fibrin clot

Cytokines/chemokines
Malignant epithelial cells

Figure 1.

Wound healing versus invasive tumour growth. a, Normal tissues have a highly organized and segregated architecture. Epithelial cells sit atop a basement membrane separated from the vascularized stromal (dermis) compartment. Upon wounding or tissue assault, platelets are activated and form a haemostatic plug where they release vasoactive mediators that regulate vascular permeability, influx of serum fibrinogen, and formation of the fibrin clot. Chemotactic factors such as transforming growth factor- $\beta$ and platelet-derived growth factor, derived from activated platelets, initiate granulation tissue formation, activation of fibroblasts, and induction and activation of proteolytic enzymes necessary for remodelling of the extracellular matrix (for example, matrix metalloproteinases and urokinase-type plasminogen activator). In combination, granulocytes, monocytes and fibroblasts are recruited, the venous network restored, and re-epithelialization across the wound occurs. Epithelial and stromal cell types engage in a reciprocal signalling dialogue to facilitate healing. Once the wound is healed, the reciprocal signalling subsides. b. Invasive carcinomas are less organized. Neoplasia-associated angiogenesis and lymphangiogenesis produces a chaotic vascular organization of blood vessels and lymphatics where neoplastic cells interact with other cell types (mesenchymal,

haematopoietic and lymphoid) and a remodelled extracellular matrix. Although the vascular network is not disrupted in the same way during neoplastic progression as it is during wounding, many reciprocal interactions occur in parallel. Neoplastic cells produce an array of cytokines and chemokines that are mitogenic and/or chemoattractants for granulocytes, mast cells, monocytes/macrophages, fibroblasts and endothelial cells. In addition, activated fibroblasts and infiltrating inflammatory cells secrete proteolytic enzymes, cytokines and chemokines, which are mitogenic for neoplastic cells, as well as endothelial cells involved in neoangiogenesis and lymphangiogenesis. These factors potentiate tumour growth, stimulate angiogenesis, induce fibroblast migration and maturation, and enable metastatic spread via engagement with either the venous or lymphatic networks. 


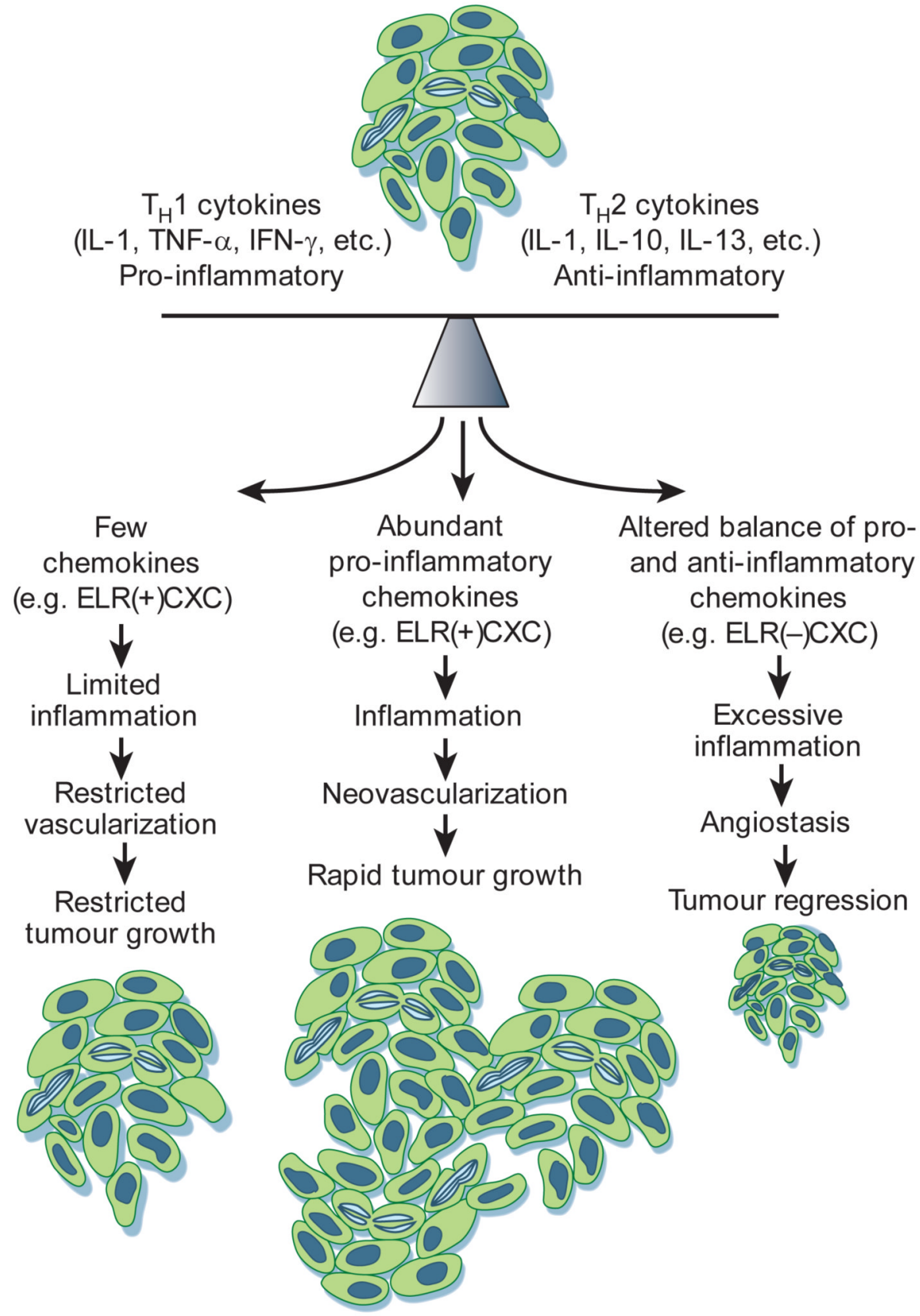

Figure 2.

Cytokine and chemokine balances regulate neoplastic outcome. The balance of cytokines in any given tumour is critical for regulating the type and extent of inflammatory infiltrate that forms. Tumours that produce little or no cytokines or an overabundance of anti-inflammatory cytokines induce limited inflammatory and vascular responses, resulting in constrained tumour growth. In contrast, production of an abundance of pro-inflammatory cytokines can lead to a level of inflammation that potentiates angiogenesis, thus favouring neoplastic growth.

Alternatively, high levels of monocytes and/or neutrophil infiltration, in response to an altered balance of pro-versus anti-inflammatory cytokines, can be associated with cytotoxicity, 
angiostasis and tumour regression. In tumours, interleukin-10 is generally a product of tumour cells and tumour-associated macrophages. 


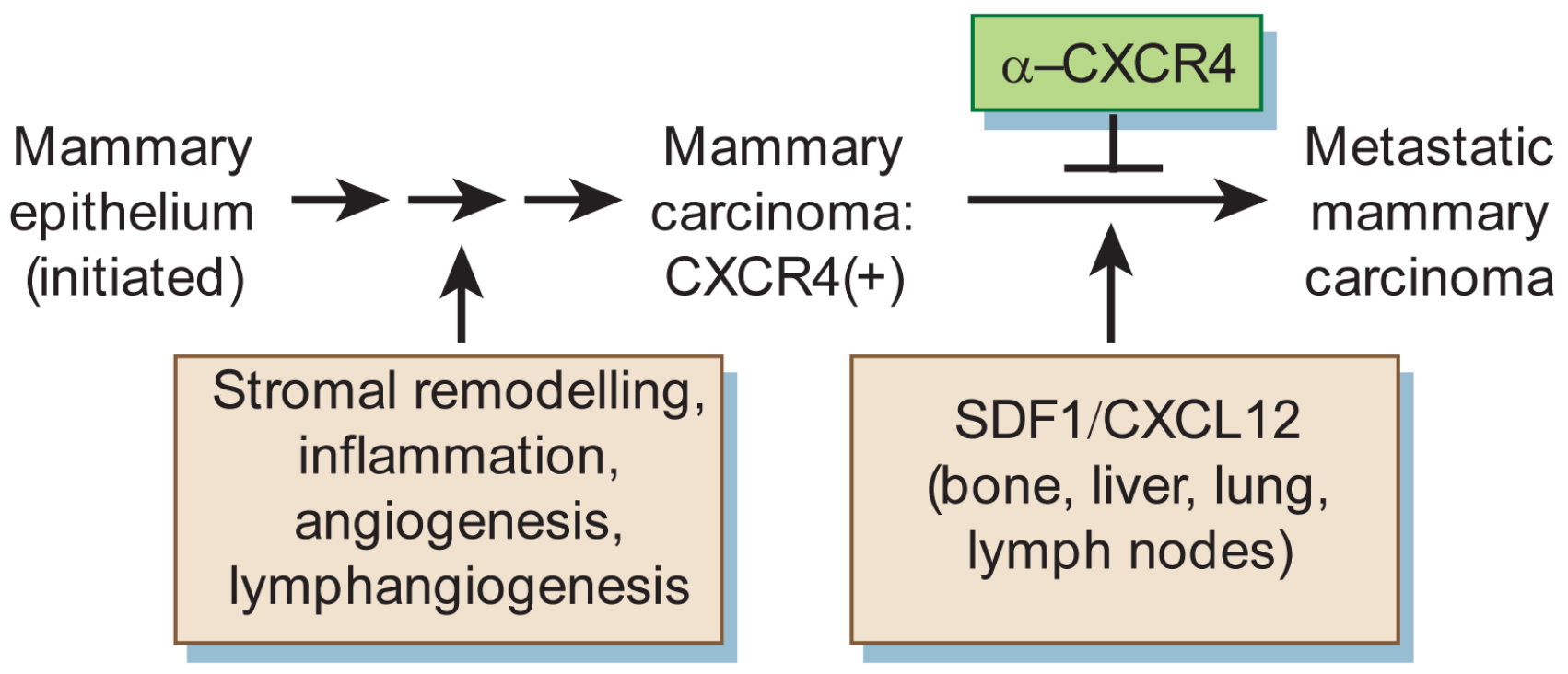

Figure 3.

Cancer metastasis and chemokine signalling. Initiated epithelial cells are promoted by inflammation to undergo neoplastic progression, a process that requires remodelling of the extracellular matrix, recruitment of inflammatory cells, angiogenesis and lymphangiogenesis. Out of this microenvironment, carcinomas arise. These neoplastic cells then turn on expression of chemokine receptors, such as CXCR4. The production of chemokine ligands for these receptors, in sites such as lymph nodes, bone marrow, liver and lung, then facilitates their invasion and migration to secondary sites where malignant cells reside either in a dormant state, or proliferate to form a productive metastatic lesion. Blockade of chemokine receptors, for example, anti-CXCR4 antibodies, attenuates metastatic spread in some experimental systems. 
Table 1

Chronic inflammatory conditions associated with neoplasms

\begin{tabular}{|c|c|c|}
\hline Pathologic condition & Associated neoplasm(s) & Aetiologic agent \\
\hline Asbestosis, silicosis & Mesothelioma, lung carcinoma & $\begin{array}{l}\text { Asbestos fibres, silica } \\
\text { particles }\end{array}$ \\
\hline Bronchitis & Lung carcinoma & $\begin{array}{l}\text { Silica, asbestos, smoking } \\
\text { (nitrosamines, peroxides) }\end{array}$ \\
\hline Cystitis, bladder inflammation & Bladder carcinoma & $\begin{array}{l}\text { Chronic indwelling, } \\
\text { urinary catheters }\end{array}$ \\
\hline Gingivitis, lichen planus & Oral squamous cell carcinoma & \\
\hline $\begin{array}{l}\text { Inflammatory bowel disease, } \\
\text { Crohn's disease, chronic ulcerative } \\
\text { colitis }\end{array}$ & Colorectal carcinoma & \\
\hline Lichen sclerosus & Vulvar squamous cell carcinoma & \\
\hline $\begin{array}{l}\text { Chronic pancreatitis, hereditary } \\
\text { pancreatitis }\end{array}$ & Pancreatic carcinoma & $\begin{array}{l}\text { Alcholism, mutation in } \\
\text { trypsinogen gene on } \mathrm{Ch} \text {. } \\
7\end{array}$ \\
\hline $\begin{array}{l}\text { Reflux oesophagitis, Barrett's } \\
\text { oesophagus }\end{array}$ & Oesophageal carcinoma & Gastric acids \\
\hline Sialadenitis & Salivary gland carcinoma & \\
\hline $\begin{array}{l}\text { Sjögren syndrome, Hashimoto's } \\
\text { thyroiditis }\end{array}$ & MALT lymphoma & \\
\hline Skin inflammation & Melanoma & Ultraviolet light \\
\hline \multicolumn{3}{|c|}{ Cancers associated with infectious agents } \\
\hline Opisthorchis, Cholangitis & Cholangiosarcoma, colon carcnoma & $\begin{array}{l}\text { Liver flukes } \\
\text { (Opisthorchis viverrini), } \\
\text { bile acids }\end{array}$ \\
\hline Chronic cholecystitis & Gall bladder cancer & $\begin{array}{l}\text { Bacteria, gall bladder } \\
\text { stones }\end{array}$ \\
\hline Gastritis/ulcers & Gastric adenocarcinoma, MALT & Helicobacter pylori \\
\hline Hepatitis & Hepatocellular carcinoma & Hepatitis B and/or C virus \\
\hline Mononucleosis & $\begin{array}{l}\text { B-cell non-Hodgkin's lymphoma, Burkitts } \\
\text { lymphoma, }\end{array}$ & Epstein-Barr Virus \\
\hline AIDS & $\begin{array}{l}\text { Non-Hodgkin's lymphoma, squamous cell } \\
\text { carcinomas, Kaposi's sarcoma }\end{array}$ & $\begin{array}{l}\text { Human } \\
\text { immunodeficiency virus, } \\
\text { human herpesvirus type } 8\end{array}$ \\
\hline Osteomyelitis & Skin carcinoma in draining sinuses & Bacterial infection \\
\hline $\begin{array}{l}\text { Pelvic inflammatory disease, chronic } \\
\text { cervicitis }\end{array}$ & $\begin{array}{l}\text { Ovarian carcinoma, cervical/anal } \\
\text { carcinoma }\end{array}$ & $\begin{array}{l}\text { Gonnorrhoea, chlamydia, } \\
\text { human papillomavirus }\end{array}$ \\
\hline Chronic cystitis & $\begin{array}{l}\text { Bladder, liver, rectal carcinoma, follicular } \\
\text { lymphoma of the spleen }\end{array}$ & Schistosomiasis \\
\hline
\end{tabular}

Modified from refs 29, 67. MALT, mucosa-associated lymphoid tissue. 